\title{
To Lump or Split When Assessing Psoriatic Arthritis - Not Mutually Exclusive?
}
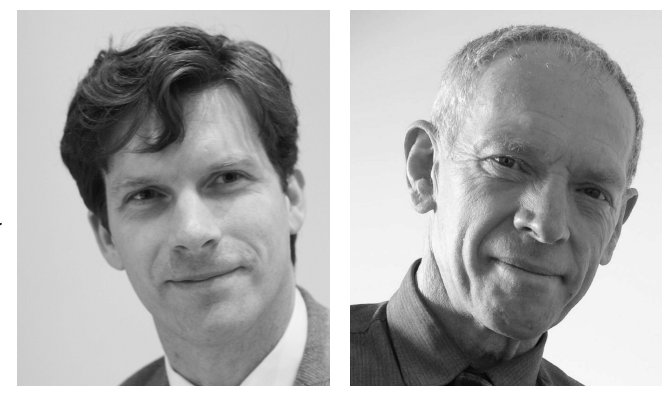

Arguably, the most important advances in modern clinical care have arisen not through the development of new drugs but instead through a recognition that the Gestalt approach to disease assessment is simply not adequate when it comes to selecting and assessing response to therapy. Accurate and regular assessment with an appropriate measurement instrument gives the clinician and patient reliable information to track disease trajectory and make treatment decisions. Achieving consensus on a single disease-specific instrument has considerable advantages, facilitating adoption of international treatment guidelines, and interpretation of data from trials, cohorts, and registries to make translation into routine care seamless ${ }^{1}$. In the context of rheumatoid arthritis (RA), the 28-joint count Disease Activity Score (DAS28) has been widely adopted as the most frequently used generally continuous measure of activity. The DAS28 has established cutpoints for high, moderate, and low disease activity and remission. The term near remission is increasingly preferred because it better differentiates those with some residual disease from those in true remission. Clinicians are used to the measure and what the numbers mean clinically, and this has allowed a smooth translation of research findings into clinical practice, including the implementation of treat to target and adoption of clinical guidelines. It has been harder to achieve this consensus in the field of psoriatic arthritis (PsA), with no current agreement on the most appropriate instrument to adopt. Why is this the case, and what are the key barriers ${ }^{2}$ ?

The greatest challenge in the adoption of a composite measure for routine care in PsA has been philosophical: should we incorporate multiple domains of disease into a single measure to identify the totality of disease, or should we focus on 1 domain at a time for accurate assessment and to avoid diluting responsiveness? PsA may manifest in a variety of different ways, with combinations of peripheral joint disease, skin psoriasis, enthesitis, dactylitis, and axial disease. The argument for "lumping" domains together has been the desire to record the totality of disease in a single numeric value. There is a perceived need for this in PsA because peripheral joint disease is generally less destructive than RA but the effect of disease on quality of life and work is similar, because of the accumulation of disease activity in multiple domains. It is only through the incorporation of multiple domains of disease into a single composite measure that we can quantify the total level of disease. A number of candidate composite measures have been developed to record multiple domains of disease, including (but not limited to) the Psoriatic Arthritis Disease Activity Score (PASDAS), the Composite Psoriatic Disease Activity Index (CPDAI), and the GRAPPA Composite Exercise (GRACE) ${ }^{3}$ index. The concern with lumping domains together is that it may not be philosophically desirable to condense such diverse aspects of a disease into a single numerical value. A further disadvantage may be that a resulting score may be less responsive and may remain static if one aspect or domain of disease improves while the other deteriorates. The Disease Activity Index for Psoriatic Arthritis (DAPSA) score is largely an articular measure [comprising a 66/68 joint count, patient pain and global activity ratings, and C-reactive protein (CRP)] and thus it can be argued that it is 1-dimensional ${ }^{4}$. If the DAPSA is used, other domains of disease need to be evaluated separately. While the approach of focusing on articular disease in a single measure avoids the issue of lumping domains together, the concern has been that the DAPSA will underestimate the totality of disease activity by focusing solely on peripheral articular disease. To add to this debate, Perruccio and colleagues provide further evidence in this issue of The Journal; so, what do their data contribute ${ }^{5}$ ?

The PASDAS is an example of a continuous composite measure of disease activity in PsA as opposed to a response criterion such as achieving minimal disease activity (MDA), which is a binary state that you are either in or not ${ }^{6,7}$. The PASDAS is an 8-item score composed of the 66 swollen and 68 tender joint counts, physician and patient global visual analog scales (VAS), Leeds Enthesitis Index, tender dactylitis count, physical function component of the Medical Outcomes Study Short Form-36 questionnaire (or SF-12), and $\mathrm{CRP}^{8}$. The final PASDAS score is derived from a

See PASDAS and MDA in PsA, page 362

Personal non-commercial use only. The Journal of Rheumatology Copyright (C) 2020. All rights reserved. 
weighted formula that gives a single numeric value of disease activity; the score ranges between 0 (no disease) and 10 (severe disease). The mathematical model included only outcomes that optimized the ability of the PASDAS to detect change; therefore the PASDAS is not an "all-inclusive" or comprehensive composite - for example, the skin is excluded. This approach has parallels with the DAS28, which does not include assessment of the feet in RA because the foot joints were found not to contribute any extra information.

The aim of the study by Perruccio, et $a l^{5}$ was to externally validate the high, moderate, and low PASDAS disease activity states, as well as to define cutoffs for near remission and low disease activity. Clinical and patient-reported outcomes were collected from 178 patients attending the University of Toronto PsA clinic. The PASDAS, MDA (a state of low disease activity), and very low disease activity (VLDA; 7 out of 7 MDA, as a state of near remission) were subsequently calculated. Receiver-operating characteristic curve (ROC) analysis identified a PASDAS score of 3.2 and 2.1 maximized the sensitivity and specificity for MDA and VLDA, respectively. These estimates correlate well with the analyses from the GRACE dataset (PASDAS 3.2 for LDA and 1.9 for VLDA), which gives external validity to previously defined cutoffs (Figure 1). The study is elegantly simple with few limitations to its interpretation. The cohort is representative of those commonly seen in rheumatology clinics. The mean disease duration was well established at 17 years. However, a fifth of those included had disease duration of $<5$ years, giving confidence that the results are generalizable across the spectrum of disease duration. Similarly, it is desirable that a composite score should perform well among those with oligoarticular as well as polyarticular disease, and $15 \%$ of participants had oligoarticular disease, which is representative of the proportion seen in clinical practice. So how should we interpret these data, and should we now use the PASDAS in routine practice?

The PASDAS was developed specifically for PsA in the GRACE study (as opposed to borrowed and adapted from another disease) and so has good face validity; there is also evidence for its reliability, feasibility, and responsiveness in trial and observational cohort studies ${ }^{3,9,10}$. Perhaps unsurprisingly, given its method of development, the PASDAS outperforms other composite measures in randomized controlled trials and observational datasets and predicts radiographic progression ${ }^{11,12}$. With the new data from Perruccio, et $a l^{5}$ in this issue of The Journal there is now have external validation of clinically relevant cutpoints for disease activity ${ }^{7}$. Subsequent analysis proposed that a PASDAS score of 1.9 or less corresponded with near remission as defined by VLDA ${ }^{13}$. The study by Perruccio, et al now provides external validation for these clinically relevant disease states for use in the clinical setting. Therefore, it seems the only barrier to the wider adoption of the PASDAS remains the philosophical question we posed in the title of this editorial: should we lump outcomes together (as in the PASDAS) or split them (and measure individual domains separately, as has been done in the DAPSA)? We suggest that the two are not mutually exclusive and it is helpful to look to the DAS28 again to explain why. As clinicians, when we use the DAS28 in RA we assess the joints, global VAS, and CRP to get a global measure of disease, but we examine the individual elements as they are recorded. If there are no swollen joints and the CRP is normal, we instinctively interpret the numeric value differently and consider imaging and assessing for other causes of pain. It is second nature to treating clinicians to interpret the component parts of the DAS28 in addition to the total score ${ }^{14}$. So the single numeric value of a composite score is only interpreted in the context of its component parts; in other words, the component parts of a composite measure and final score are not mutually exclusive.

The core purpose of a composite measure of disease activity is to provide a measure of disease state in a single numeric value that has clinical meaning. Such a composite score has greater power in discerning outcomes and gives us a metric by which to convey information to our colleagues and patients. Further, in a disease such as PsA, the individual components themselves may not rate as severe (by any metric, including the insurance companies and government healthcare comissioners), yet the composite score may achieve that description. For the PASDAS, the data from the study by Perruccio, et al give external validity to the clinically relevant disease states of low disease and near

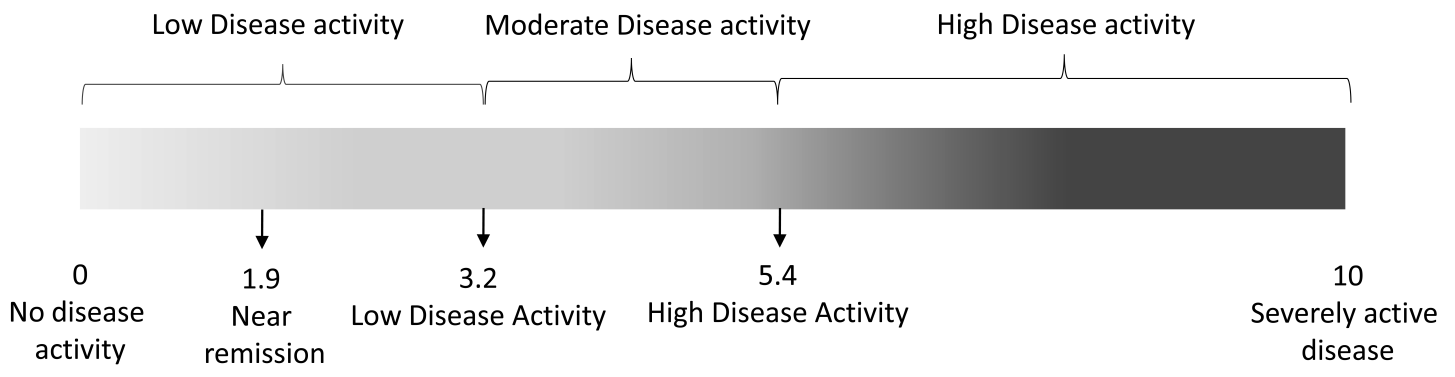

Figure 1. Clinically relevant cutpoints for the Psoriatic Arthritis Disease Activity Score. Near remission is equivalent to very low disease activity. Low disease activity is equivalent to minimal disease activity.

Personal non-commercial use only. The Journal of Rheumatology Copyright (C) 2020. All rights reserved 
remission, which can be applied in clinical practice ${ }^{5}$. By using the PASDAS as we have become used to using the DAS28, by clinically reflecting on the component parts as the score is calculated, we also preserve the advantages of lumping outcomes together, without the philosophical disadvantages.

\section{WILLIAM TILLETT (iD), $\mathrm{MBChB}^{\mathrm{PhD} \text {, }}$} Royal National Hospital for Rheumatic Diseases, Bath;

PHILIP HELLIWELL (iD), MD, PhD, Chapel Allerton Hospital, Leeds, UK.

Address correspondence to W. Tillett, Royal National Hospital for Rheumatic Diseases, Upper Borough Walls, Bath BA1 1RL, UK. E-mail:w.tillett@nhs.net

\section{REFERENCES}

1. Tillett W, Orbai AM, Ogdie A, Leung YY, Strand V, Gladman DD, et al. GRAPPA-OMERACT initiative to standardise outcomes in psoriatic arthritis clinical trials and longitudinal observational studies. Ann Rheum Dis 2018;77:e23.

2. Coates LC, FitzGerald O, Merola JF, Smolen J, van Mens LJ, Bertheussen H, et al. Group for Research and Assessment of Psoriasis and Psoriatic Arthritis/Outcome Measures in Rheumatology Consensus-Based Recommendations and Research Agenda for Use of Composite Measures and Treatment Targets in Psoriatic Arthritis. Arthritis Rheumatol 2018;70:345-55.

3. Helliwell PS, FitzGerald O, Fransen J. Composite disease activity and responder indices for psoriatic arthritis: a report from the GRAPPA 2013 meeting on development of cutoffs for both disease activity states and response. J Rheumatol 2014;41:1212-7.

4. Schoels MM, Aletaha D, Alasti F, Smolen JS. Disease activity in psoriatic arthritis (PsA): defining remission and treatment success using the DAPSA score. Ann Rheum Dis 2016;75:811-8.

5. Perruccio AV, Got M, Li S, Ye Y, Gladman DD, Chandran V. Treating psoriatic arthritis to target: defining the psoriatic arthritis disease activity score that reflects a state of minimal disease activity. J Rheumatol 2020;47:362-8.

6. Helliwell PS, FitzGerald O, Fransen J, Gladman DD, Kreuger GG, Callis-Duffin K, et al. The development of candidate composite disease activity and responder indices for psoriatic arthritis (GRACE project). Ann Rheum Dis 2013;72:986-91.

7. Helliwell PS, Kavanaugh A. Comparison of composite measures of disease activity in psoriatic arthritis using data from an interventional study with golimumab. Arthritis Care Res 2014;66:749-56.

8. Helliwell PS, Waxman R. Modification of the Psoriatic Arthritis Disease Activity Score (PASDAS). Ann Rheum Dis 2018;77:467-8.

9. Mulder M, den Broeder A, van Ginneken B, Vriezekolk J, Mahler E, van den Hoogen F, et al. Implementing the Psoriatic Arthritis Disease Activity Score (PASDAS) in routine clinical practice: (im)possible? Ann Rheum Dis 2019;78 Suppl 2:A1282.

10. Coates LC, Merola JF, Fitzgerald O, Kavanaugh A, Gottlieb AB, Tillett W, et al. AB0742 Achievement of PASDAS low disease activity and very low disease activity in patients with psoriatic arthritis treated with certolizumab pegol over 4 years and the overlap with DAPSA and MDA disease activity targets [abstract]. BMJ 2019;78 Suppl 2.

11. Helliwell PS, Kavanaugh A. Radiographic progression in psoriatic arthritis achieving a good response to treatment: data using newer composite indices of disease activity. Arthritis Care Res 2018;70:797-800.

12. Mease P, Gladman D, Collier D, Ritchlin C, Helliwell P, Liu L, et al. Etanercept and methotrexate as monotherapy or in combination for psoriatic arthritis: primary results from a randomized, controlled phase III trial. Arthritis Rheumatol 2019;71:1112-24.

13. Coates LC, Helliwell PS. Defining low disease activity states in psoriatic arthritis using novel composite disease instruments. J Rheumatol 2016;43:371-5.

14. Lindsay K, Ibrahim G, Sokoll K, Tripathi M, Melsom RD, Helliwell PS. The composite DAS Score is impractical to use in daily practice: evidence that physicians use the objective component of the DAS in decision making. J Clin Rheumatol 2009;15:223-5.

J Rheumatol 2020;47:307-9; doi:10.3899/jrheum.190867 